\title{
Functional Mock-up Interface in Mechatronic Gearshift Simulation for Commercial Vehicles
}

\author{
Andreas Abel $^{2} \quad$ Torsten Blochwitz ${ }^{2} \quad$ Alexander Eichberger ${ }^{3}$ \\ Peter Hamann ${ }^{1} \quad$ Udo Rein $^{1}$ \\ ${ }^{1}$ Daimler AG, HPC B209, 70546 Stuttgart, Germany \\ ${ }^{2}$ ITI GmbH, Webergasse 1, 01067 Dresden, Germany \\ ${ }^{3}$ SIMPACK AG, Friedrichshafener Str. 1, 82205 Gilching, Germany \\ Abel@itisim.com, Blochwitz@itisim.com, Alex.Eichberger@SIMPACK.de, \\ Peter.Hamann@daimler.com, Udo.Rein@daimler.com
}

\begin{abstract}
Mechatronic shifting simulation of automated transmissions in commercial vehicles is used for optimization and development in today's truck engineering departments at Daimler. Within the ITEA2 project Modelisar in cooperation with ITI GmbH and SIMPACK AG this application served as a usecase for proof of concept of the newly developed Functional Mock-Up interfaces (FMI). Utilizing these standardized interfaces models from different tools are coupled to build up the overall system for the mechatronic shifting simulation. The coupling via FMI for Model Exchange was achieved for control modules from MATLAB/Simulink into the SimulationX powertrain model and secondly from the 1Dmultiphysics powertrain in SimulationX into multibody vehicle in SIMPACK. Furthermore FMI for Co-Simulation was investigated in a pure SimulationX framework for the powertrain model. Very promising results can be observed as for modeling as for simulation processes. The FMI technology has clearly shown its capability to be applied in the productive simulation process.
\end{abstract}

Keywords: FMI, Modelisar, multibody system, automated shifting, mechatronics, co-simulation, model exchange

\section{Introduction}

The ITEA2 project Modelisar was a European research initiative from 2008 till 2011 focusing on the overall development process "from System Modeling to S/W running on the Vehicle". The major outcome is the standardization proposal Functional Mock-Up Interface (FMI) to facilitate tool and model coupling on implementation and numerical level, e.g. see [4], [5]. Within the project several usecases served as proof of concept by utilizing tools providing the new interfaces.

One usecase was the "Mechatronic shifting simulation of commercial vehicles" provided by Daimler AG, ITI GmbH and SIMPACK AG. This workpackage uses a Functional Mock-Up (FMU) of an automated gear shift system within the truck powertrain focusing on the transmission and demonstrating several benefits of the Modelisar environment. The SiL simulation is used for optimizing gear shift times and shifting comfort in heavy-duty trucks. Major challenges are the number of sub-models from different simulation tools and the necessary standardization of modeling, coupling, and solving.

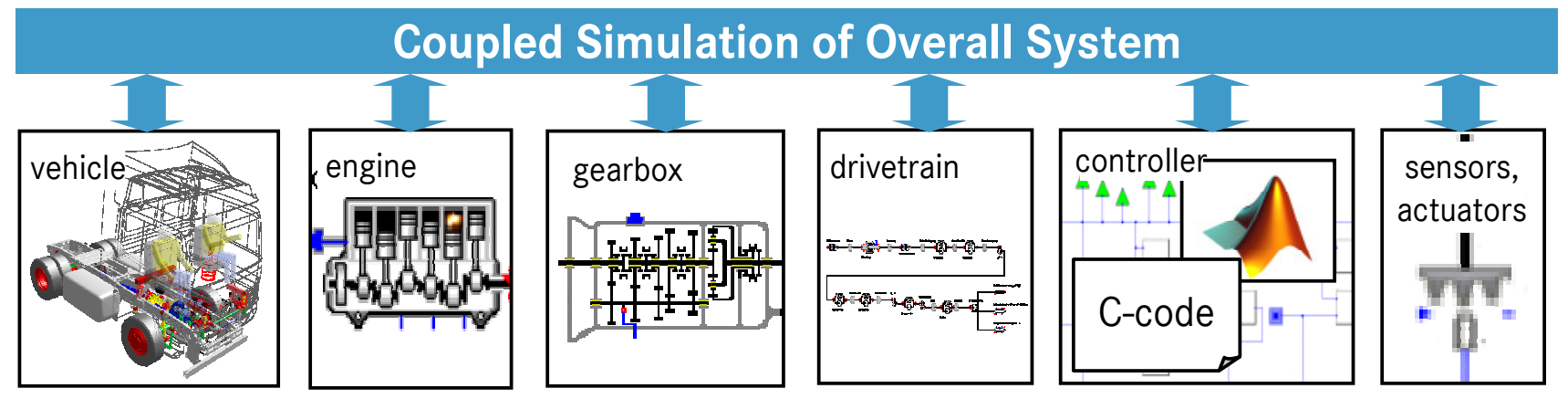

Fig 1.1 - FMU Mechatronic Shifting Simulation 


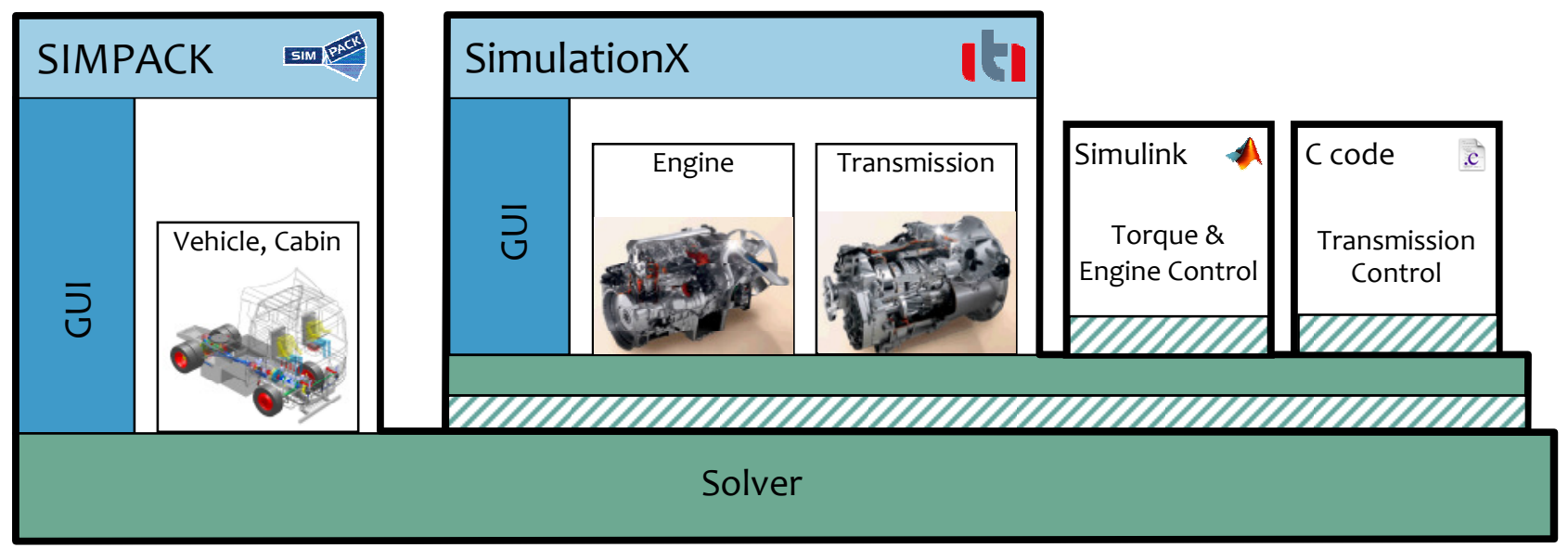

Fig 2.1 - FMI for Model Exchange Usecase Prototype

\section{Mechatronic shifting simulation}

\subsection{The Modelisar usecase}

The demonstration target for the Modelisar usecase has been a fully shiftable powertrain of heavy-duty trucks, which models the physics and control structure of the overall vehicle in such a degree of detail, that all phases of a gear shift can be reflected in terms of interactions between the driveline and vehicle dynamics, and the different control units participating in the shift. The models are capable to treat the large-scale low-frequency effects such as driveline jerking, as well as high-frequency phenomena, such as dynamics of actuation systems and gearbox components, engine combustion, or the impact of CAN bus delays on the overall system behavior.

The model allows the accurate prediction of the performance of the different driveline control units in interaction with the truck, the vehicle responses, and the perception of the driveline operation by the driver in terms of driving and shifting comfort.

This type of modeling requires the integration of a heterogeneous collection of models created for various simulation environments:

- 1D torsional vibration models of engine, gearbox - modeled in SimulationX ${ }^{\circledR 1}$,

- Actuation systems on clutch and gearbox modeled in SimulationX,

- Detailed multibody (mbs) vehicle - modeled in SIMPACK $^{\circledR 2}$

\footnotetext{
${ }^{1}$ SimulationX is a registered trademark of ITI GmbH

${ }^{2}$ SIMPACK is a registered trademark of SIMPACK AG
}

- Controller model from external supplier - compiled C code generated from MATLAB/ Simulink

- DAIMLER in-house controller code - C code and MATLAB/Simulink models,

- Re-engineered controller functionality where no appropriate source was available - modeled in Modelica ${ }^{\circledR 3}$ within SimulationX.

Thus, this application has been a perfect target within the MODELISAR project to verify technologies developed in the project framework, for the FMI as well as for FMI-based co-simulation technologies.

The main objectives of the usecase were decreased simulation time, improved processes and overall decreased development times utilizing the FMI coupling techniques. Therefore the Simulationin-the-Loop (SiL) implementations are based on the FMI-enhanced new versions of SimulationX, SIMPACK and MATLAB/Simulink representing control functionalities and powertrain models.

\subsection{Simulation environment at project start}

The usecase started with a simulation framework as shown in Figure 2.2, see [1], [2]. It included already all necessary models for the mechatronic shifting simulation. The coupling was based on proprietary non-standard interfaces from SimulationX and SIMPACK.

\footnotetext{
${ }^{3}$ Modelica is a registered trademark of the Modelica Association
} 
1D powertrain model (SimulationX)

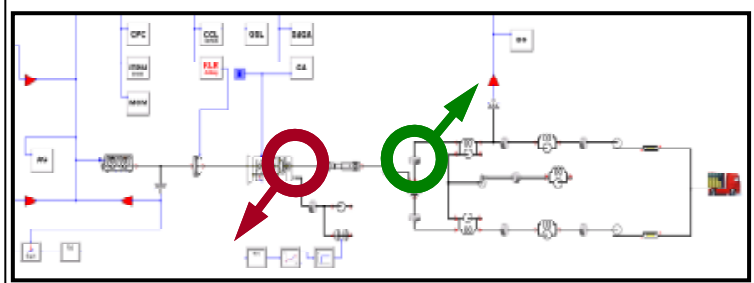

The control unit was integrated into the SimulationX powertrain model utilizing Modelica external functions on one hand via MATLAB ${ }^{\circledR} /$ Simulink $^{\circledR}$ and a Real-Time Workshop ${ }^{\circledR 4}$ (RTW) SimulationX target or the other hand via wrapped exported CCode. The resulting time excitations stimulated the vehicle model in SIMPACK as an offline coupling.

Although this simulation environment already delivered detailed and qualitatively good results many reasons for improvement were observed: The offline coupling omits any feedback of powertrain and vehicle. The model exchange was as well for the software modules as for the powertrain module mainly handwritten and error prone, inefficient and costly to maintain.

\section{FMI for Model Exchange}

Within this use case the FMI for Model Exchange has been the main instrument to achieve the desired tool interoperation and model transfers. Using the FMI for Model Exchange controllers have been connected to a SimulationX driveline model, which in turn has been integrated into SIMPACK using the very same technology, see [3].

\subsection{Control unit integration}

Control software development for engine, clutch and gearbox control for Daimler commercial vehicles is done mainly inhouse. Software development stretches over a wide and dynamic range of MATLAB/Simulink and TargetLink ${ }^{\circledR 5}$ versions but also includes plain $\mathrm{C}$ code. Thus achieving a software-inthe-loop (SiL) integration of these control unit mod-

\footnotetext{
${ }^{4}$ MATLAB, Simulink and Real-Time Workshop are registered trademarks of The MathWorks, Inc.

${ }^{5}$ TargetLink is a trademark of dSPACE GmbH
}

ules provides a certain challenge but has also a high potential to be facilitated with a standardized interface such as FMI. Within the usecase FMI applicability has been examined in two variants:

Variant 1 applies to controllers implemented in MATLAB/Simulink and utilizes a RTW toolbox provided by Dassault Systèmes AB through another Modelisar work package in order to export the Simulink model as FMU. Such FMU can be integrated easily into SimulationX. So this variant was the primary path in the usecase to verify the FMI interface.

Variant 2 implemented an FMI wrapper for plain controller $\mathrm{C}$ code. This was tested only prototypically within Modelisar using small test models. The approach has been demonstrated to be applicable but the absence of an automated FMI wrapper generation (e.g. through scripting) and the necessary degree of manual preparation of the FMU so far does not allow a productive use in the overall simulation process.

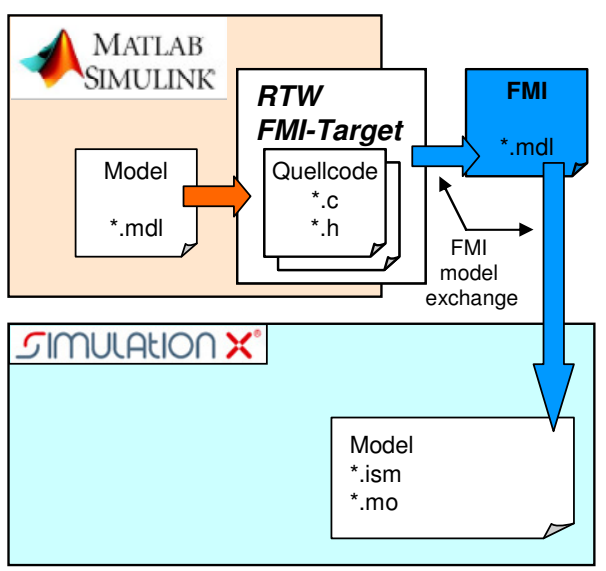

Fig 3.1 - Process chain for transferring MATLAB/ Simulink controller models into SimulationX

The migration to FMI based SiL controller models has been achieved within the productive simulation process by gradually replacing the tool-specific solutions based on a dedicated SimulationX target for the Real-Time Workshop (RTW). 


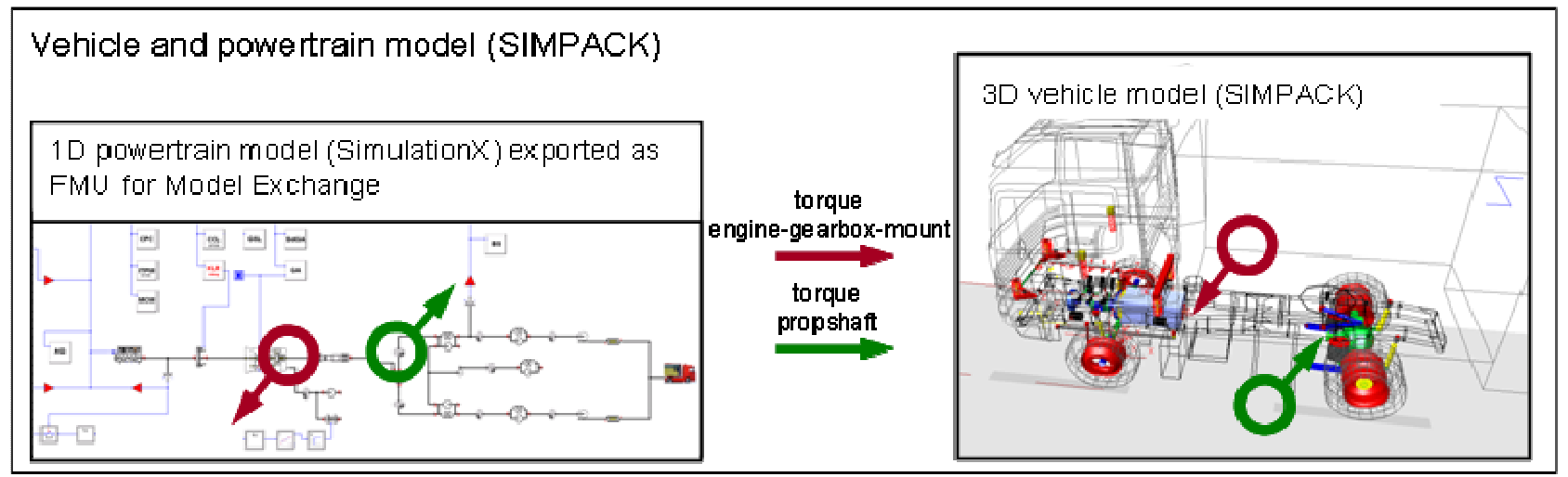

Fig 3.2 - FMI without feedback

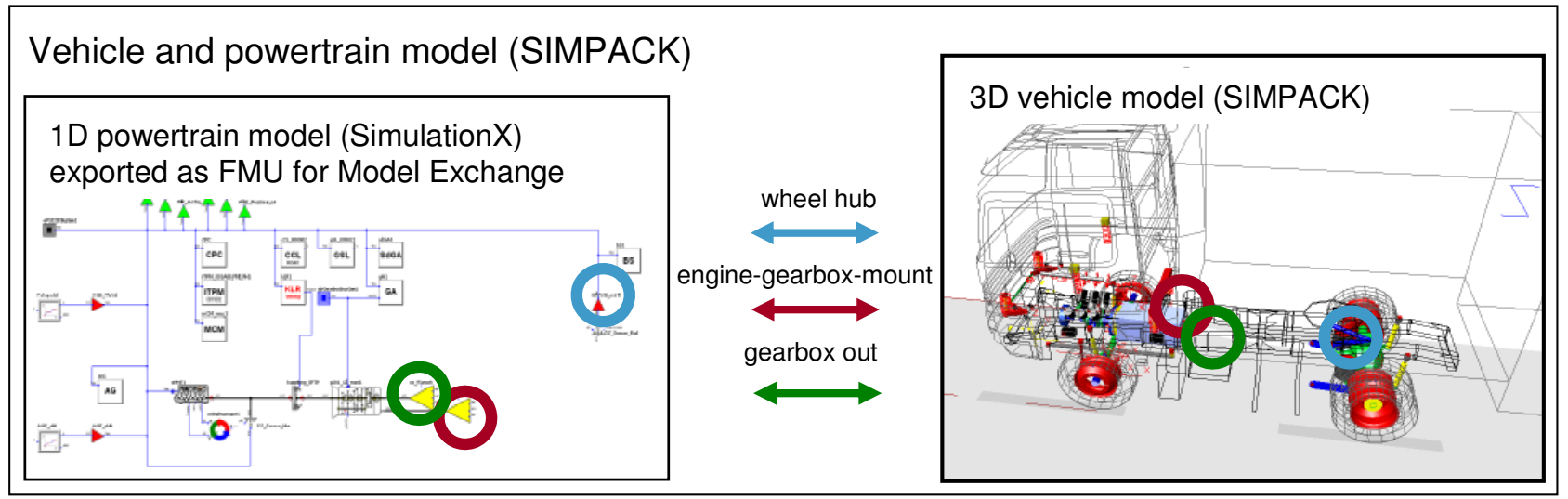

Fig 3.3 - FMI with feedback

Applying the standardized FMI technology now allows a higher degree of automation in the model exchange process and significantly broadens the range of potential target environments for the mechatronic gearshift simulation. The application of FMI also did not affect results and simulation performance.

\subsection{FMU import without feedback}

In a first stage prototype, the powertrain FMU including the control unit of section 3.1 was imported by FMI in the SIMPACK vehicle model. Rather than using offline pre-calculated inputs to the mbs-model as shown in section 2.2, online generated inputs where used in the simulation (see Figure 3.2).

Cutting point of the SIMPACK powertrain model is between the first cardan shaft and the differential on the rear axle. All rotational parts in front of the cardan joint are modeled as 1-D model within SimulationX and imported via FMI in SIMPACK. All rotational parts behind the cardan joint and the complex vehicle 3D-structure are modeled in SIMPACK.

No input from SIMPACK is passed into the FMU during simulation (without feedback).
FMU output, being applied to SIMPACK as kinetic excitations:

- torque on the gearbox output,

- torque on the rear differential input.

The results of the FMU integration without feedback show a perfect match with the results of the approach of section 2.2. The SIMPACK solver step size of the SIMPACK vehicle model without FMU was approximately 1e-3s. By integrating the FMU in the SIMPACK model, the overall simulation time increased due to the limiting step size of 20e-6s induced by the FMU. The integration time can potentially be reduced by a co-simulation between these two models, see section 4. Another approach is a performance optimization of the powertrain model in SimulationX. First model analyses show a high potential to at least gain an acceleration of factor 10 .

\subsection{FMU import with feedback - full FMI for Model Exchange solution}

In the final stage prototype, the dynamic feedback between powertrain FMU and vehicle model was taken into account (see Figure 3.3)

Cutting point of the SIMPACK powertrain model is behind the gearbox output flange. All rotational parts in front of this flange are modeled as 1D model 


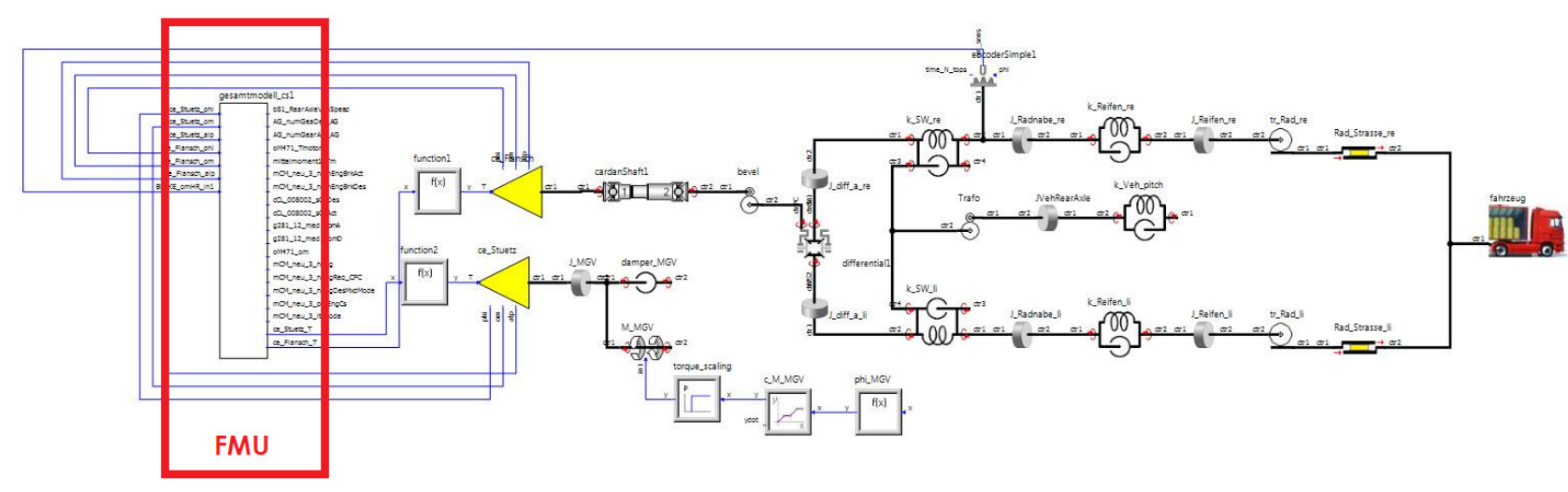

\section{Fig 4.1 - FMI for Co-Simulation with Vehicle Model in SimulationX}

within SimulationX and imported via FMI in SIMPACK. All rotational parts behind this flange and the complex vehicle 3D-structure are modeled in SIMPACK.

The FMU uses the following values as input (with feedback), being kinematic measurements of SIMPACK:

- relative angle of the engine block wrt. vehicle frame,

- relative rot. velocity of the engine block wrt. vehicle frame,

- relative angle of the front propeller shaft flange wrt. engine block,

- relative rot. velocity of the front propeller shaft flange wrt. engine block,

- angle of the rear wheels.

FMU output, being applied to SIMPACK as kinetic excitations:

- torque on the gearbox output,

- torque on the front propeller shaft flange.

The feedback introduces a new level of accuracy for simulation of shifting comfort and increases simulation quality at Daimler.

The simulation performance shows the same characteristics as described in chapter 3.2. The step size is dominated completely by the high dynamic powertrain model and no additional difficulties due to the feedback are introduced. The future work will focus on FMI for Co-Simulation on one hand and performance increase in the powertrain model on the other hand.

\section{FMI for Co-Simulation}

Sharing models between different simulation tools using the FMI for Model Exchange potentially provides pitfalls if the tools are using different solver technologies or if models run on significantly different time scales. The latter is also the case in the model coupling between SimulationX and SIMPACK.

The FMI for Co-Simulation is a potential solution since it allows bundling a model with a dedicated solver, which can run independently of the solver in the target system. On the other hand a co-simulation between models of physically coupled sub-systems may be difficult due to the inherently introduced communication time delays in the coupling.

During the usecase project the FMI for CoSimulation in SIMPACK still has been under development. For this reason an alternative test scenario has been implemented, where the FMI of the driveline which is intended for SIMPACK integration has been re-imported into SimulationX and has been coupled with a model of the downstream driveline (from the differential onwards), see [3]. In terms of present natural frequencies and discontinuities this reflects a similar scenario as in SIMPACK, where the vehicle part of the model could be solved with significantly bigger time steps.

The maximum achievable stepsize in the communication and the impact of different interpolation methods between communication steps have been assessed. This test case showed a clear need to balance the communication stepsize in order to achieve stable and valid simulation results. Although the communication for a physical link still needs very small step sizes, the communication stepsizes are about 10 times larger than the required stepsizes using an FMI for Model Exchange. This allows expecting an equivalent 10 -fold performance increase.

\section{Conclusions and Outlook}

The usecase Mechatronic Shifting Simulation was completed successfully by implementing an FMIbased simulation framework already improving the development at Daimler and furthermore showing high potential for a wider future use. The new interface standardization proposals FMI for Model Ex- 
change and FMI for Co-Simulation were implemented in different prototypes arising in the field of drivetrain dynamics and especially mechatronic shifting simulation for commercial vehicles.

To model and simulate the mechatronic powertrain SimulationX is used. Since version 3.4 SimulationX implements FMI for Model Exchange as import and export, as well as FMI for Co-Simulation as Slave and Master. All four variants have been tested successfully in different prototypes.

The vehicle and cabin is modeled as $3 \mathrm{D}$ multibody system in SIMPACK. The version SIMPACK 9.0 implements FMI for Model Exchange as import. This interface has been tested successfully for different prototypical implementations.

Furthermore the control unit software had to be imported from MATLAB/Simulink. This could be achieved successfully via FMI for Model Exchange with the RTW toolbox as well developed within Modelisar.

From a technical point of view the following result could be achieved:

- Prototypical FMI based simulation of mechatronic shifting system with bi-directional coupling of vehicle and powertrain model,

- FMI based model exchange of MATLAB/Simulink control modules into powertrain model in SimulationX,

- FMI based model exchange of SimulationX powertrain model into SIMPACK vehicle model, but simulation performance needs further improvement due to problem specific multi-scale behavior,

- Alternative bi-directional coupling via FMI for Co-Simulation started.

FMI makes coupling of models easier to implement. The numerics of the coupling regarding performance, model harmonization, etc. must be analyzed as before. The potential of FMI for CoSimulation could be shown. As soon as implementations are available it will be investigated for the coupling of vehicle and powertrain model for the mechatronic shifting simulation.

In the result of the Modelisar project the further development and improvement of the FMI standard has become a core task within the Modelica community. Due to the high industrial acceptance and feasibility proven by projects such as the presented use case, tool vendors eagerly follow these developments within their tools.

SIMPACK Version 9 officially supports Model Import based on FMI Standard 1.0. Co-Simulation based on FMI Standard 1.0 is currently under development and will be available fall 2012.
ITI has been driving FMI developments from the very beginning and fully supports all FMI variants in SimulationX since 2010.

As soon as FMI Standard 2.0 has been officially released, SIMPACK and ITI are going to upgrade the FMI interfaces in their tools to this version.

To summarize it can be said that with the new coupling interfaces enormous benefits for industrial applications can be generated: They reduce significantly the implementation complexity and costs for tool and model coupling. They optimize the SiL processes regarding time, cost, robustness and quality. They simplify internal and external model exchange and model reuse.

\section{References}

[1] A. Abel (ITI GmbH), P. Hamann, U. Rein (Daimler AG) Modelisar: sWP303 Mechatronic shifting simulation, Milestone report: Documentation of specification and simulation environment, ITEA2 Modelisar internal paper, 2009

[2] A. Abel (ITI GmbH), P. Hamann, U. Rein (Daimler AG) Modelisar: sWP303 Mechatronic shifting simulation, Milestone report: Results prototype 1, ITEA2 Modelisar internal paper, 2010

[3] A. Abel, T. Blochwitz (ITI GmbH), M. Friedrich, J. Zeman (SIMPACK AG), P. Hamann, U. Rein (Daimler AG) Modelisar: sWP303 Mechatronic shifting simulation, Milestone report: Results for FMI prototype, ITEA2 Modelisar internal paper, 2011

[4] T. Blochwitz, T. Neidhold (ITI GmbH), M. Otter (DLR), M. Arnold (University of Halle), C. Bausch, M. Monteiro (Atego Systems $\mathrm{GmbH}$ ), C. Claus, S. Wolf (Fraunhofer IIS, EAS), H. Elmqvist, H. Olsson (Dassault Systemes), A. Junghanns, J. Mauss (QTronic GmbH), D. Neumerkel (Daimler AG), J. Peetz (Fraunhofer SCAI): The Functional Mock-Up Interface, Modelica Confererence 2011, Dresden

[5] FMI-Homepage including FMI specifications www.fmi-standard.org 